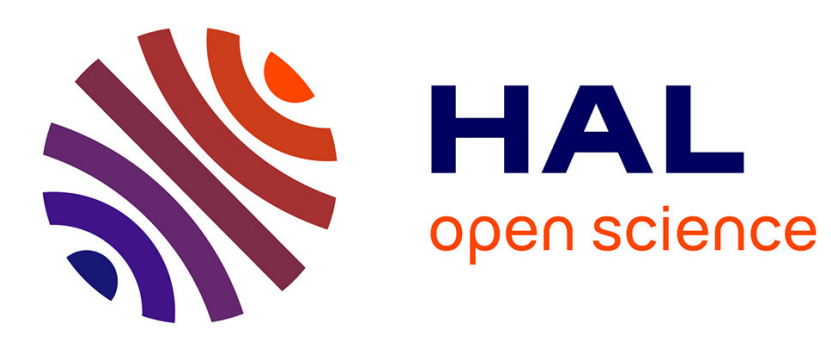

\title{
Efficient method for RCS computation of long and complex dielectric objects
}

Saul Perez, M'Barek Fares, Bernard Souny

\section{To cite this version:}

Saul Perez, M'Barek Fares, Bernard Souny. Efficient method for RCS computation of long and complex dielectric objects. EuMW 2006, 36th European Microwave Conference, Sep 2006, Manchester, United Kingdom. pp 1300-1303, 10.1109/EUMC.2006.281235 . hal-01021783

\section{HAL Id: hal-01021783 \\ https://hal-enac.archives-ouvertes.fr/hal-01021783}

Submitted on 25 Sep 2014

HAL is a multi-disciplinary open access archive for the deposit and dissemination of scientific research documents, whether they are published or not. The documents may come from teaching and research institutions in France or abroad, or from public or private research centers.
L'archive ouverte pluridisciplinaire HAL, est destinée au dépôt et à la diffusion de documents scientifiques de niveau recherche, publiés ou non, émanant des établissements d'enseignement et de recherche français ou étrangers, des laboratoires publics ou privés. 


\title{
Efficient for RCS computation of long and complex dielectric objects
}

\author{
Saul Perez ${ }^{1}$, M'Barek Fares $^{2}$, and Bernard Souny ${ }^{3}$ \\ ${ }^{1}$ ENAC, Toulouse, perez@ recherche.enac.fr, France (7, Avenue Edouard Belin - BP 4005 31055) \\ ${ }^{2}$ CERFACS, Toulouse, fares@ cerfacs.fr, France (42, Avenue G. Coriolis - 31057) \\ ${ }^{3}$ ENAC, Toulouse, souny @ recherche.enac.fr, France (7, Avenue Edouard Belin - BP 400531055$)$
}

\begin{abstract}
We propose a new method for RCS computation of long internally complex dielectric objects such as wind turbine blades. The method proposed here is an improved iterative algorithm whose convergence is proven. Thanks to the geometrical decomposition technique, three accelerating methods can be used efficiently in an iterative algorithm. The first accelerating method allows the elimination of the internal degrees of freedom with minimal cost. The second accelerating method (matrix compression $Q R)$ accelerates all the matrix vector products used in the preconditioning procedure as well as in the GMRES iterative resolution. The third accelerating method consists in using a "geometric neighboring" preconditioner adapted to the physical aspect of the problem.
\end{abstract}

Index Terms - Integral equations, Method of Moment, Parallel algorithm, Iterative methods.

\section{INTRODUCTION}

We propose a new method for Radar Cross Section (RCS) computation of long internally complex, dielectric objects such as wind turbine blades. RCS computation of these objects is important for the aviation community to determine the wind farms impact on radio navigation systems. These objects are far greater than the wavelength but asymptotic methods cannot be applied to solve this type of complex problem. The method of moment (MoM) is a popular choice for solving electromagnetic scattering problems by an arbitrary object. However it is well known that the traditional Method of Method (MoM) suffers from the storage requirement (increasing in the order of $O\left(N^{2}\right)$ ) and computational complexity (increasing in the order of $\left.O\left(N^{3}\right)\right)$ for large scale problems.

These limitations impose on the one hand the use of a cluster to share out the storage and the computational complexity cost and on the other hand the use of an iterative method (increasing in the order of $O\left(N^{2}\right)$ ) to reduce the computational complexity cost.

A solution frequently explored to share out the storage requirement and the computational complexity is the resolution proposed by the method called Region by Region [1, 2, 3]. The method formulates the problem into operator blocks, corresponding to geometrical groups and are filled using the traditional MoM taken from [4]. The iterative algorithm identifies with the block Jacobi methods. It is well known that these iterative pose a mathematical problem because the convergence is not proven within the MoM framework [2]. So Krylov iterative solvers were investigated with different preconditioning techniques combined with accelerating methods to resolve large problems.

The methods proposed here is an improved iterative algorithm whose convergence is proven. Moreover the algorithm can be easily adapted to parallel computation. We use the same formulation as in the Region by Region method. Thanks to the geometrical decomposition technique, three accelerating methods can be used efficiently in an iterative algorithm. The first accelerating method allows the elimination of the internal degrees of freedom that do not participate in RCS computation. The second accelerating method (matrix compression QR) accelerates all the matrix vector products used in the preconditioning procedure (reduction from $O\left(N^{2}\right)$ to $\left.O\left(N^{i .5}\right)\right)$ as well as in the GMRES iterative resolution (reduction from $O\left(N^{2}\right)$ to $O\left(N^{i .5}\right)$ ). This accelerating method also reduces the memory requirement (reduction from $O\left(N^{2}\right)$ to $\left.O\left(N^{l 5}\right)\right)$. The simplicity of the implementation in an existing code is another advantage of this method in comparison with fast mutipole methods (FMM). The third accelerating method consists in using a "geometric-neighboring" preconditioner adapted to the physical aspect of the problem. Thus the formulation per block and the mathematical tools used (accelerating method and iterative method) form a robust iterative algorithm which is adapted to parallel computation.

\section{DESCRIPTION OF THE METHOD}

Fig. 1 represents a simple model of a wind turbine blade used to validate our algorithm. Validation is performed comparing our solution with the direct solution given by Cerfacs Electromagnetic Solver Code (CESC) [4]. 
We explain below the geometrical decomposition technique and its the advantage for each step in our algorithm.

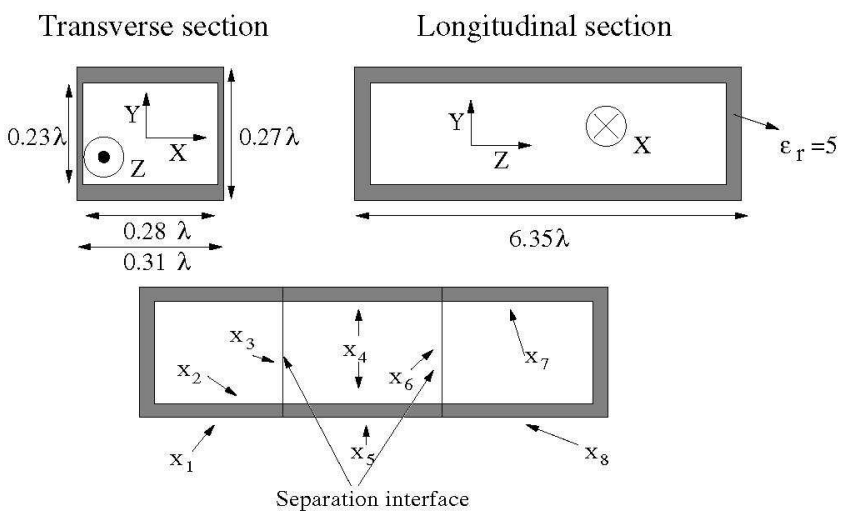

Fig. 1

\section{A. Advantage of the decomposition technique for internal degrees elimination}

The internal degrees of freedom do not participate in RCS computation. Their elimination reduces the initial system, which saves memory and resolution time. This technique is particularly interesting for long internally complex objects.

The proposed geometrical decomposition technique introduces a separation interface which divides the simple model into several sections. Separation interfaces introduce an additional and moderate number of degrees of freedom, but this technique leads to a high reduction of CPU time.

In the formulation used operator blocks $Z_{\mathrm{i}, \mathrm{j}}$ correspond to the radiating operators originated by the basis functions $X_{i}$ (residing on the block $\mathrm{i}$ ) and tested by weighting functions $T_{j}$ (residing on the block $j$ ). We can easily demonstrate that $Z_{\mathrm{i}, j \neq\left\{\mathrm{k}_{\}}\right\}}=0$ and $Z_{\mathrm{j} \neq\left\{\mathrm{k}_{\}}, 1\right.}=0$ if block $\mathrm{i}$ is surrounded by a closed surface composed by the blocks $\{\mathrm{k}\}$, where $\mathrm{j}$ is an exterior block. The separation interfaces create internal blocks $\left(\mathrm{X}_{2}, \mathrm{X}_{4}, \mathrm{X}_{7}\right)$ surrounded by a closed surface (Fig. 1), thus introducing many null operators in the linear system.

In order to understand the consequences of these null operators we describe step by step the elimination of internal degrees of freedom $\mathrm{X}_{2}$ (Fig. 1).

The elimination of internal degrees is a successive elimination procedure. The first step consists in expressing the internal unknown $\mathrm{X}_{2}$ in terms of the other unknowns of the problem and thanks to the property exposed:

$$
Z_{2,(j \neq 1,2,3)}=0 \text { and } X_{2}=Z_{22}^{-1}\left[\sum_{i=1, i \neq 2}^{3} Z_{2, i} X_{i}+S_{2}\right]
$$

Where $\mathrm{S}_{2}$ is the known excitation tested by weighting functions $\mathrm{T}_{2}$.

The second step consists in introducing the equation (1) in the remaining equations of the linear system, thanks to the same property $Z_{(j=1,2,3), 2}=0$. thus the equation (1) will be introduced only in equations 1 and 3 . Finally we obtain a new linear system where $X_{2}$ has disappeared. This methodology allows us to greatly the number of the matrix-matrix products in the elimination of internal degrees of freedom.

The degrees of freedom introduced by separation interfaces could be eliminated for the same reasons as internal degrees of freedom. But the number of matrixmatrix products increases rapidly as one proceeds with the successive elimination of these degrees of freedom. Consequently the unknowns residing on the separation interfaces are conserved.

The Tab1 shows the reduction of unknowns eliminated in the case of a $6.35 \lambda$ long simple model of a wind turbine blade. This simple model is decomposed into many blocks and the mesh size is about $\lambda / 5$.

\begin{tabular}{|l|c|c|c|c|}
\hline Number of blocks & 2 & 4 & 8 & 16 \\
\hline Initial unknowns & 3182 & 3578 & 4370 & 5954 \\
\hline $\begin{array}{l}\text { unknowns after } \\
\text { elimination }\end{array}$ & 1918 & 2202 & 2770 & 3906 \\
\hline Reduction rate & $36 \%$ & $38 \%$ & $40 \%$ & $43 \%$ \\
\hline
\end{tabular}

Tab 1

\section{B. Advantage of the decomposition technique for the} preconditioning procedure

The decomposition technique allows us to define a simple "geometric neighboring" preconditioner such as a block Jacobi preconditioner which is easily set up. Moreover this preconditioner can be easily integrated into parallel computation. Spectral analysis for canonical examples show that this type of preconditioner accurately models ill-conditioning due to the high frequency terms, but does not capture the long range interactions [5]. If we extrapolate the conclusions in [5] in our case we can expect that the condition number scales as $\mathrm{D}^{0.5}$, where $\mathrm{D}$ is the block size. Fig.2 shows the increase of the number iterations is lower than expected.

Indeed "geometric neighboring" preconditioner has an interesting property illustrated in Fig.2: the number of iterations increases slowly when the number of fixed size blocks increases. The increase of the number of iterations is slower when the block is large. 
For all our simulations the preconditioner allows us to obtain such a well-conditioned linear system that GMRES converges in only a few iterations without restart.

We may expect to obtain a well-conditioned linear system for a large by using a large block size. The size of the geometrical is fixed by memory available. The resolution of large problems is achieved by increasing the number of blocks.

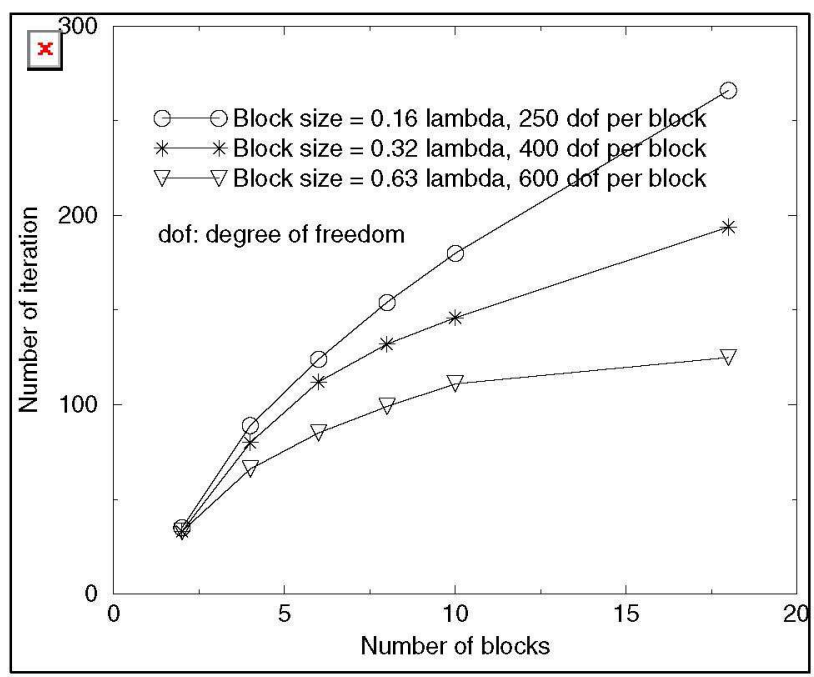

Fig. 2

C. Advantage of the decomposition technique for compression of the matrix operators

The decomposition technique leads to the definition of concentrated geometrical blocks which are more or less distant (Fig. 1). When two blocks are well-separated relatively to their size, the rank of the interaction block operator decreases [6]. We make use of the rank deficiency of the off-diagonal operators $Z_{i, j \neq i}$ to accelerate matrix vector products.

We proceed to a matrix compression by QR factorisation using a compressing criterion $\mathrm{E}_{\mathrm{C}}$ equivalent to the one proposed in [7]: the rank of matrix $Q$ and $R$ is $k$ if $k$ is the larger number such as $\mathrm{R}(\mathrm{k}+1, \mathrm{k}+1) \leq \mathrm{E}_{\mathrm{C}} \times \mathrm{R}(1,1)$.

The approximation introduced by compression amplifies numeric errors at the final solution when the linear system is ill-conditioned [8]. It would be better to compress the off-diagonal operators after the preconditioning procedure. By spectral analyses it is possible to demonstrate that the approximation affects the high frequency currents. In our case, we deal with RCS computation and the radiated high frequency waves are evanescent which almost vanish at the distance where we calculate the RCS. So, we have decided to compress the matrices before the preconditioning procedure to accelerate computation.

We give in Fig. 3 numerical results for the $6.35 \lambda$ long simple model decomposed into 8 sections for different compress criterions $\mathrm{E}_{\mathrm{C}}$. The bistatic RCS is computed at $\varphi=0$ and $\theta=[0, \pi]$. We compare RCS computed by our algorithm with the numerical results given by Cerfacs Electromagnetism Solver Code (CESC) [4]. Fig. 3 shows that $E_{C}=1 \mathrm{e}-6$ is sufficiently for the RCS computation.

In the case where $E_{C}=1 e-6$ we have observed the reduction of computation complexity in the preconditioning procedure (reduction from $O\left(N^{2}\right)$ to $O\left(N^{1.5}\right)$ ). The matrix compression also reduces the computation complexity per iteration (reduction from $O\left(N^{2}\right)$ to $\left.O\left(N^{1.5}\right)\right)$ and the storage requirement (reduction from $O\left(N^{2}\right)$ to $O\left(N^{1.5}\right)$ ).

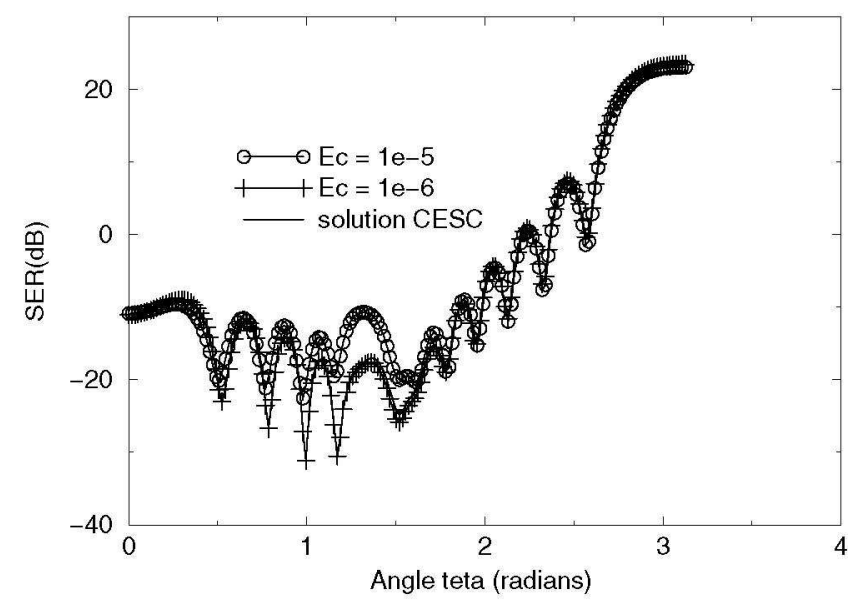

Fig. 3

\section{CONCLUSION}

The combination of different techniques discussed in this article allows us to obtain an efficient method for resolution of scattering problems by long internally complex, dielectric objects.

Indeed association of the elimination of internal degrees of freedom, the compression $\mathrm{QR}$ technique and the 'geometric-neighboring' preconditioner based on the physical aspects of the problem have allowed to reduce the storage memory requirement and the computational complexity. We can expect to resolve RCS computation problems of real long complex objects such as a real wind turbine blade.

\section{REFERENCES}

[1] Connor Brennan, Peter Cullen, and Marissa Condon, "A Novel Iterative Solution of the Three Dimensional Electric Field Integral Equation", IEEE Trans. Antennas Propragat., Vol. 52, pp. 2781-2784, October 2004.

[2] Véronique Tirel, "Hybridation par méthode de décomposition de bord appliquées à l'étude des ondes acoustiques et électromagnétiques, résultats théoriques et numériques", thèse Paris X7II, 1998.

[3] Abir Atig, "Etude du rayonnement d'une source placée sur un corps métallique de forme arbitraire ", thèse INPT 2001. 
[4] A. Bendali, C. Devys, "Calcul numérique du rayonnement de cornets électromagnétiques dont l'ouverture est partiellement remplie par diélectrique'", Rapport Polytechnique 1982.

[5] Karl F. Warnick and Wend Cho Chew, "On the spectrum of the electric field integral equation and the convergence of the moment method", International Journal for numerical methods in engineering 2001 51:31-56.

[6] Ovidio M. Bucci, Giorgio Franceschetti, "On the Degrees of Freedom of Scattered Fields", IEEE Trans. Antennas Propragat., Vol. 37 N7, pp. 918-926, July 1989.
[7] Gene H. Golub, Charles F. Van Loan, 'Matrix Computations'.

[8] Jean René Poirier, "Modélisation électromagnétique des effets de rugosité surfacique", thèse INSA 2000. 